\title{
Quantum effects on KG-oscillator field under a Cornell-type potential in Kaluza-Klein theory
}

\author{
Faizuddin Ahmed ${ }^{1}$ \\ National Academy Gauripur, 783331, Assam, India
}

\begin{abstract}
In this paper, we solve KG-oscillator in the five-dimensional cosmic string space-time background with a uniform magnetic field and quantum flux subject to a scalar potential of Cornell-type using KaluzaKlein theory, and observe the gravitational analogue of the AharonovBohm effect. We show that the energy eigenvalue and eigenfunction depends on the global parameters of the space-time, and also a quantum effect is seen due to the dependence of magnetic field on the quantum numbers of the system.
\end{abstract}

keywords: Kaluza-Klein theory, Relativistic wave equation, AharonovBohm effect.

PACS Number: 03.65.-w, 03.65.Pm, 03.65.Ge, 11.27.+d, 04.50.Cd,

\section{Introduction}

The Klein-Gordon (KG) oscillator $[1,2]$ was inspired by earlier papers on the Dirac oscillator [3] applied to half-integer spin particles. Several authors have studied the Klein-Gordon oscillator, for example, in Ref. [4] Klein-Gordon oscillator in the background space-time generated by the cosmic string in the presence of a uniform magnetic field, in Ref. [5] Klein-Gordon oscillator in the presence of a Coulomb potential, in Ref. [6] quantum effects of confining potentials on Klein-Gordon oscillator, in Ref. [7] Klein-Gordon oscillator in

\footnotetext{
${ }^{1}$ faizuddinahmed15@gmail.com ; faiz4U.enter@rediffmail.com
} 
the Som-Raychaudhuri space-time in the presence of a uniform magnetic field , in Ref. [8] Klein-Gordon oscillator in the background space-time generated by a cosmic string, in Ref. [9] Klein-Gordon oscillator on the curved background within the Kaluza-Klein theory, in Ref. [10] Klein-Gordon oscillator in the background space-time generated by a rotating cosmic string subject to a Cornell-type scalar and Coulomb-type vector potentials including an internal magnetic flux, in Ref. [11] Klein-Gordon oscillator in the cosmic string space-time with a spacelike dislocation subject to a scalar potential, in Ref. [12] generalized Klein-Gordon oscillator field on the background space-time induced by a spinning cosmic string coupled to a homogeneous magnetic field including a magnetic quantum flux, in Ref. [13] generalized Klein-Gordon oscillator in cosmic string space-time with a scalar potential of Cornell-type within the Kaluza-Klein theory, in Ref. [14] generalized Klein-Gordon oscillator in the cosmic string space-time with a linear scalar potential within the Kaluza-Klein theory, in Ref. [15] Klein-Gordon oscillator in the presence of an external field including magnetic quantum flux subject to a Cornell-type scalar potential in cosmic string with a space-like dislocation, and in Ref. [16] Klein-Gordon oscillator in a topologically trivial linear class of Gödel-type space-time has been studied.

In this work, we study a Klein-Gordon oscillator in 5D space-time background produced by topological defects within the Kaluza-Klein theory (KKT) $[17,18,19]$. Topological defects play an important role in condensed matter physics systems $[20,21,22,23,24]$ where, topological defects analogue to cosmic strings appear in phase transitions in liquid crystals [25, 26]. Geometric quantum phases [27] describe the phase shifts acquire by wave-function of a quantum mechanical particle. A well-known quantum phase is the AharonovBohm effect $[28,29,30]$ due to the presence of an internal magnetic flux produced by the topological defects space-times. This effect has investigated in, Newtonian theory [31], bound states of massive fermions [32], scattering of dislocated wave-fronts [33], torsion effects on a position-dependent mass 
system [11, 15, 34, 35, 36, 37, 38], bound states solution of spin-0 scalar particles in different space-times geometry background [10, 12, 39, 40]. In addition, this effect has investigated within the Kaluza-Klein theory by introducing scalar potentials of various kind by several authors in the relativistic quantum mechanics [9, 13, 14, 41, 42, 43, 44, 45, 46].

In Ref. [47], interaction between KG-oscillator and scalar potential of different kinds in the five-dimensional Minkowski space-time background using Kaluza-Klein theory was investigated. In this paper, we study the same problem in the background of the five-dimensional cosmic string space-time with a uniform magnetic field using Kaluza-Klein theory. We see a quantum effect due to the dependence of the magnetic field on the quantum numbers $\{n, l\}$ of the quantum system. Also we see that the energy level shifted due to the presence of the cosmic string parameter in comparison to those result obtained in the five-dimensional Minkowski space-time in [47].

\section{KG-oscillator in a magnetic cosmic string space-time with scalar potential in KKT}

In the context of Kaluza-Klein theory [17, 18], the metric with a magnetic quantum flux $(\Phi)$ passing along the symmetry axis of the string assumes the following form

$$
d s^{2}=-d t^{2}+d r^{2}+\alpha^{2} r^{2} d \phi^{2}+d z^{2}+\left[d y+\kappa A_{\mu}\left(x^{\mu}\right) d x^{\mu}\right]^{2},
$$

where $\mu=0,1,2,3, t=x^{0}$ is the time-coordinate, $y=x^{4}$ is the coordinate associated with fifth additional dimension having ranges $0<y<2 \pi a$ where, $a$ is the radius of the compact dimension of $y, 0 \leq r\left(=x^{1}\right)<\infty, 0 \leq \phi(=$ $\left.x^{2}\right)<2 \pi \alpha,-\infty<z\left(=x^{3}\right)<\infty$, and $\kappa$ is the Kaluza constant [41]. The parameter $\alpha=(1-4 \mu)$ [48] characterizing the wedge parameter where, $\mu$ is the linear mass density of the string. In gravitation and cosmology, we assume the values of the parameter $\alpha$ are in the ranges $0<\alpha \leq 1$. 
Based on $[41,43,9,13,14]$, we introduce a uniform magnetic field $B_{0}$ and magnetic quantum flux $\Phi$ through the line-element of the cosmic string space-time (1) in the following form

$$
d s^{2}=-d t^{2}+d r^{2}+\alpha^{2} r^{2} d \phi^{2}+d z^{2}+\left[d y+\left(-\frac{1}{2} \alpha B_{0} r^{2}+\frac{\Phi}{2 \pi}\right) d \phi\right]^{2},
$$

where the gauge field given by

$$
A_{\phi}=\kappa^{-1}\left(-\frac{1}{2} \alpha B_{0} r^{2}+\frac{\Phi}{2 \pi}\right)
$$

gives rise to a uniform magnetic field $\vec{B}=\vec{\nabla} \times \vec{A}=-\kappa^{-1} B_{0} \hat{z}[49], \hat{z}$ is the unitary vector in the $z$-direction. Here $\Phi=$ const is the magnetic quantum flux $[30,49]$ through the core of the topological defects [50].

The relativistic quantum dynamics of spin-0 scalar particle with a scalar potential $S(r)$ in five-dimensions is described by [34, 15, 13]:

$$
\left[\frac{1}{\sqrt{-g}} \partial_{M}\left(\sqrt{-g} g^{M N} \partial_{N}\right)-(m+S)^{2}\right] \Psi=0
$$

where $M, N=0,1,2,3,4$ with $g$ is the determinant of the metric tensor with $g^{M N}$ its inverse.

To couple oscillator with the KG-field, we change the following radial momentum operator

$$
\vec{p} \rightarrow \vec{p}+i m \Omega r \hat{r} \quad, \quad \partial_{r} \rightarrow \partial_{r}+m \Omega r,
$$

such that $\vec{p}^{2} \rightarrow(\vec{p}+i m \Omega r \hat{r})(\vec{p}-i m \Omega r \hat{r})$.

Therefore, the KG-equation becomes

$$
\left[\frac{1}{\sqrt{-g}}\left(\partial_{M}+m \Omega r\right) \sqrt{-g} g^{M N}\left(\partial_{N}-m \Omega r\right)-(m+S)^{2}\right] \Psi=0,
$$

For the metric (2)

$$
g^{M N}=\left(\begin{array}{ccccc}
-1 & 0 & 0 & 0 & 0 \\
0 & 1 & 0 & 0 & 0 \\
0 & 0 & \frac{1}{\alpha^{2} r^{2}} & 0 & -\frac{\kappa A_{\phi}}{\alpha^{2} r^{2}} \\
0 & 0 & 0 & 1 & 0 \\
0 & 0 & -\frac{\kappa A_{\phi}}{\alpha^{2} r^{2}} & 0 & 1+\frac{\kappa^{2} A_{\phi}^{2}}{\alpha^{2} r^{2}}
\end{array}\right) .
$$


By considering the line-element (2) into the Eq. (6), we obtain the following differential equation :

$$
\begin{aligned}
& {\left[-\frac{\partial^{2}}{\partial t^{2}}+\frac{\partial^{2}}{\partial r^{2}}+\frac{1}{r} \frac{\partial}{\partial r}+\frac{1}{\alpha^{2} r^{2}}\left(\frac{\partial}{\partial \phi}-\kappa A_{\phi} \frac{\partial}{\partial y}\right)^{2}+\frac{\partial^{2}}{\partial z^{2}}+\frac{\partial^{2}}{\partial y^{2}}\right.} \\
& \left.-2 m \Omega-m^{2} \Omega^{2} r^{2}-(m+S)^{2}\right] \Psi(t, r, \phi, z)=0 .
\end{aligned}
$$

Since the line-element (2) is independent of $t, \phi, z, y$. One can choose the following ansatz for the function $\Psi$ as:

$$
\Psi(t, r, \phi, z, y)=e^{i(-E t+l \phi+k z+q y)} \psi(r)
$$

where $E$ is the total energy of the particle, $l=0, \pm 1, \pm 2, . . \in \mathbf{Z}$, and $k, q$ are constants.

Substituting the ansatz (9) into the Eq. (8), we obtain the following equation:

$\left[\frac{d^{2}}{d r^{2}}+\frac{1}{r} \frac{d}{d r}+E^{2}-k^{2}-q^{2}-2 m \Omega-m^{2} \Omega^{2} r^{2}-\frac{\left(l-\kappa q A_{\phi}\right)^{2}}{\alpha^{2} r^{2}}-(m+S)^{2}\right] \psi(r)=0$.

In this work, we choose the scalar potential of Cornell-type, and subsequently with a Coulomb-type and linear potential and obtain the bound states solution of the considered relativistic quantum system using KaluzaKlein theory.

\section{Case A : Interactions with Cornell-type potential}

The Cornell-type potential consists of linear plus Coulomb-like term is a particular case of the quark-antiquark interaction [51, 52]. The Coulomb potential is responsible at small distances or short range interactions and linear potential leads to confinement of quark. This type of potential is given by $[7,15,38]$

$$
S(r)=\frac{\eta_{c}}{r}+\eta_{L} r
$$

where $\eta_{c}, \eta_{L}$ are the potential parameters. 
Substituting eqs. (3) and (11) into the Eq. (10), we obtain the following equation:

$$
\left[\frac{d^{2}}{d r^{2}}+\frac{1}{r} \frac{d}{d r}+\lambda-\frac{j^{2}}{r^{2}}-\omega^{2} r^{2}-\frac{a}{r}-b r\right] \psi(r)=0,
$$

where

$$
\begin{aligned}
\lambda & =E^{2}-k^{2}-q^{2}-m^{2}-2 \eta_{c} \eta_{L}-2 m \omega_{c} \frac{\left(l-\frac{q \Phi}{2 \pi}\right)}{\alpha}-2 m \Omega \\
\omega & =\sqrt{m^{2} \omega_{c}^{2}+\eta_{L}^{2}+m^{2} \Omega^{2}} \\
j & =\sqrt{\frac{\left(l-\frac{q \Phi}{2 \pi}\right)^{2}}{\alpha^{2}}+\eta_{c}^{2}} \\
\omega_{c} & =\frac{q B_{0}}{2 m} \\
a & =2 m \eta_{c} \\
b & =2 m \eta_{L} .
\end{aligned}
$$

Introducing a new variable $\rho=\sqrt{\omega} r$, Eq. (12) becomes

$$
\left[\frac{d^{2}}{d \rho^{2}}+\frac{1}{\rho} \frac{d}{d \rho}+\zeta-\frac{j^{2}}{\rho^{2}}-\rho^{2}-\frac{\eta}{\rho}-\theta \rho\right] \psi(\rho)=0,
$$

where

$$
\zeta=\frac{\lambda}{\omega} \quad, \quad \eta=\frac{a}{\sqrt{\omega}} \quad, \quad \theta=\frac{b}{\omega^{\frac{3}{2}}} .
$$

Suppose the possible solution to Eq. (14) is

$$
\psi(\rho)=\rho^{j} e^{-\frac{1}{2}(\rho+\theta) \rho} H(\rho) .
$$

Substituting the solution Eq. (16) into the Eq. (14), we obtain

$$
H^{\prime \prime}(\rho)+\left[\frac{\gamma}{\rho}-\theta-2 \rho\right] H^{\prime}(\rho)+\left[-\frac{\beta}{\rho}+\Theta\right] H(\rho)=0,
$$

where

$$
\begin{aligned}
& \gamma=1+2 j, \\
& \Theta=\zeta+\frac{\theta^{2}}{4}-2(1+j), \\
& \beta=\eta+\frac{\theta}{2}(1+2 j) .
\end{aligned}
$$


Equation (17) is the biconfluent Heun's differential equation [13, 14, 15, 34, $53,54]$ and $H(\rho)$ is the Heun polynomials.

The above equation (17) can be solved by the Frobenius method. We consider the power series solution [55]

$$
H(\rho)=\sum_{i=0}^{\infty} c_{i} \rho^{i}
$$

Substituting the above power series solution into the Eq. (17), we obtain the following recurrence relation for the coefficients:

$$
c_{n+2}=\frac{1}{(n+2)(n+2+2 j)}\left[\{\beta+\theta(n+1)\} c_{n+1}-(\Theta-2 n) c_{n}\right] .
$$

And the various coefficients are

$$
\begin{aligned}
c_{1} & =\left(\frac{\eta}{\gamma}+\frac{\theta}{2}\right) c_{0}, \\
c_{2} & =\frac{1}{4(1+j)}\left[(\beta+\theta) c_{1}-\Theta c_{0}\right] .
\end{aligned}
$$

We must truncate the power series by imposing the following two conditions $[13,14,15,34,43,44,45]$ :

$$
\begin{aligned}
\Theta & =2 n, \quad(n=1,2, \ldots) \\
c_{n+1} & =0 .
\end{aligned}
$$

By analyzing the condition $\Theta=2 n$, we get the following second degree expression of the energy eigenvalues $E_{n, l}$ :

$$
\begin{aligned}
& \frac{\lambda}{\omega}+\frac{\theta^{2}}{4}-2(1+j)=2 n \\
\Rightarrow \quad & E_{n, l}= \pm\left\{k^{2}+q^{2}+m^{2}+2 \omega\left(n+1+\sqrt{\frac{\left(l-\frac{q \Phi}{2 \pi}\right)^{2}}{\alpha^{2}}+\eta_{c}^{2}}\right)\right. \\
& \left.+2 \eta_{c} \eta_{L}+2 m \omega_{c} \frac{\left(l-\frac{q \Phi}{2 \pi}\right)}{\alpha}-\frac{m^{2} \eta_{L}^{2}}{\omega^{2}}+2 m \Omega\right\}^{\frac{1}{2}}
\end{aligned}
$$


Now, we impose additional recurrence condition $c_{n+1}=0$ to find the individual energy levels and wave-functions one by one as done in $[13,14$, $15,34,56,57]$. For $n=1$, we have $\Theta=2$ and $c_{2}=0$ which implies from Eq. (21)

$$
\begin{aligned}
& c_{1}=\frac{2}{\beta+\theta} c_{0} \Rightarrow\left(\frac{\eta}{1+2 j}+\frac{\theta}{2}\right)=\frac{2}{\beta+\theta} \\
& \omega_{1, l}^{3}-\frac{a^{2}}{2(1+2 j)} \omega_{1, l}^{2}-a b\left(\frac{1+j}{1+2 j}\right) \omega_{1, l}-\frac{b^{2}}{8}(3+2 j)=0
\end{aligned}
$$

a constraint on the parameter $\omega_{1, l}$. The magnetic field $B_{0}^{1, l}$ is so adjusted that Eq. (24) can be satisfied and we have simplified by labelling:

$$
\omega_{c}^{1, l}=\frac{1}{m} \sqrt{\omega_{1, l}^{2}-\eta_{L}^{2}-m^{2} \Omega^{2}} \leftrightarrow B_{0}^{1, l}=\frac{2}{q} \sqrt{\omega_{1, l}^{2}-\eta_{L}^{2}-m^{2} \Omega^{2}} .
$$

Therefore, the ground state energy level for $n=1$ is given by

$$
\begin{aligned}
& E_{1, l}= \pm\left\{k^{2}+q^{2}+m^{2}+2 \omega_{1, l}\left(2+\sqrt{\frac{\left(l-\frac{q \Phi}{2 \pi}\right)^{2}}{\alpha^{2}}+\eta_{c}^{2}}\right)\right. \\
& \left.+2 \eta_{c} \eta_{L}+2 m \omega_{c}^{1, l} \frac{\left(l-\frac{q \Phi}{2 \pi}\right)}{\alpha}-\frac{m^{2} \eta_{L}^{2}}{\omega_{1, l}^{2}}+2 m \Omega\right\}^{\frac{1}{2}} .
\end{aligned}
$$

And the corresponding radial wave-functions is

$$
\psi_{1, l}=\rho^{\sqrt{\frac{\left(l-\frac{q \Phi}{2 \pi}\right)^{2}}{\alpha^{2}}+\eta_{c}^{2}}} e^{-\frac{1}{2}\left(\frac{2 m \eta_{L}}{\omega_{1, l}^{\frac{3}{2}}}+\rho\right) \rho}\left(c_{0}+c_{1} \rho\right),
$$

where

$$
c_{1}=\left[\frac{2 m \eta_{c}}{\sqrt{\omega_{1, l}}\left(1+2 \sqrt{\frac{\left(l-\frac{q \Phi}{2 \pi}\right)^{2}}{\alpha^{2}}+\eta_{c}^{2}}\right)}+\frac{m \eta_{L}}{\omega_{1, l}^{\frac{3}{2}}}\right] c_{0} .
$$

In Ref. [47], authors solved KG-oscillator in the five-dimensional Minkowski space-time with a quantum flux subjected to a Cornell-type potential in 
Kaluza-Klein theory (section 2). By comparing the eigenvalue and eigenfunction (26)-(28) with those results obtained in earlier, we have seen that the results presented here get modified due to the presence of a uniform magnetic field $B_{0}$ and the wedge parameter $\alpha$ whose values are in the ranges $0<\alpha \leq 1$ and break their degeneracy.

We have that, $E_{n, l}\left(\Phi+\Phi_{0}\right)=E_{n, l \mp \tau}(\Phi)$, where $\Phi_{0}= \pm \frac{2 \pi}{q} \tau$ with $\tau=$ $0,1,2, \ldots$ That means, the energy eigenvalue is a periodic function of the geometric quantum phase. This dependence of the relativistic energy level on the geometric quantum phase gives rise to a relativistic analogue of the Aharonov-Bohm effect for bound states.

So, when the particle circles the string, the total wave-function changes according to

$$
\Psi \rightarrow \Psi^{\prime}=\operatorname{Exp}\left\{\frac{2 \pi i}{\alpha}\left(l-\frac{q \Phi}{2 \pi}\right)\right\} \Psi .
$$

The angular momentum operator may be redefined as

$$
\hat{l}_{e f f}=-\frac{i}{\alpha}\left(\partial_{\phi}-\frac{\Phi}{2 \pi} \partial_{y}\right)
$$

where the additional term, $\frac{i \Phi}{2 \pi \alpha} \partial_{y}$, takes into account the Aharonov-Bohm quantum flux.

\section{Case B : Interactions with Coulomb-type potential}

We consider $\eta_{L} \rightarrow 0$ into the scalar potential $S$. Thus the Coulomb potential is given by

$$
S(r)=\frac{\eta_{c}}{r}
$$

This kind of potential has used to study position-dependent mass systems $[13,44,58,59]$ in the relativistic quantum mechanics.

The radial wave-equations Eq. (12) becomes

$$
\left[\frac{d^{2}}{d r^{2}}+\frac{1}{r} \frac{d}{d r}+\tilde{\lambda}-\frac{j^{2}}{r^{2}}-m^{2} \tilde{\omega}^{2} r^{2}-\frac{a}{r}\right] \psi(r)=0,
$$


where

$$
\begin{aligned}
& \tilde{\lambda}=E^{2}-k^{2}-q^{2}-m^{2}-2 m \omega_{c} \frac{\left(l-\frac{q \Phi}{2 \pi}\right)}{\alpha}-2 m \Omega, \\
& \tilde{\omega}=\sqrt{\omega_{c}^{2}+\Omega^{2}} .
\end{aligned}
$$

Introduce a new variable $\rho=\sqrt{m \tilde{\omega}} r$, Eq. (32) becomes

$$
\left[\frac{d^{2}}{d \rho^{2}}+\frac{1}{\rho} \frac{d}{d \rho}+\frac{\tilde{\lambda}}{m \omega}-\frac{j^{2}}{\rho^{2}}-\rho^{2}-\frac{\tilde{\eta}}{\rho}\right] \psi(\rho)=0,
$$

where $\tilde{\eta}=\frac{a}{\sqrt{m \tilde{\omega}}}$.

Suppose the possible solution to Eq. (34) is

$$
\psi(\rho)=\rho^{j} e^{-\frac{\rho^{2}}{2}} H(\rho) .
$$

Substituting the solution Eq. (16) into the Eq. (14), we obtain

$$
H^{\prime \prime}(\rho)+\left[\frac{1+2 j}{\rho}-2 \rho\right] H^{\prime}(\rho)+\left[-\frac{\tilde{\eta}}{\rho}+\tilde{\Theta}\right] H(\rho)=0,
$$

where $\tilde{\Theta}=\frac{\tilde{\lambda}}{m \tilde{\omega}}-2(1+j)$.

Equation (36) is the biconfluent Heun's differential equation [13, 14, 15, $34,53,54]$ and $H(\rho)$ is the Heun polynomials.

Substituting the power series solution (19) into the Eq. (36), we obtain the following recurrence relation for the coefficients:

$$
c_{n+2}=\frac{1}{(n+2)(n+2+2 j)}\left[\tilde{\eta} c_{n+1}-(\tilde{\Theta}-2 n) c_{n}\right] .
$$

And the various coefficients are

$$
c_{1}=\frac{\tilde{\eta}}{1+2 j} c_{0} \quad, \quad c_{2}=\frac{1}{4(1+j)}\left[\tilde{\eta} c_{1}-\Theta c_{0}\right]
$$

The power series expansion (19) becomes a polynomial of degree $n$ by imposing two conditions $[13,14,15,34,43,44,45]$ :

$$
c_{n+1}=0 \quad, \quad \tilde{\Theta}=2 n \quad(n=1,2, \ldots)
$$


By analyzing the condition $\tilde{\Theta}=2 n$, we get the following energy eigenvalues $E_{n, l}$ :

$E_{n, l}= \pm \sqrt{k^{2}+q^{2}+m^{2}+2 m \tilde{\omega}\left(n+1+\sqrt{\frac{\left(l-\frac{q \Phi}{2 \pi}\right)^{2}}{\alpha^{2}}+\eta_{c}^{2}}\right)+2 m \omega_{c} \frac{\left(l-\frac{q \Phi}{2 \pi}\right)}{\alpha}+2 m \Omega .}$

For the radial mode $n=1$, we have $\tilde{\Theta}=2$ and $c_{2}=0$ which implies

$$
\begin{aligned}
\tilde{\omega}_{1, l} & =\frac{2 m \eta_{c}^{2}}{(1+2 j)} \\
\Rightarrow \quad \omega_{c}^{1, l} & =\sqrt{\left(\frac{2 m \eta_{c}^{2}}{(1+2 j)}\right)^{2}-\Omega^{2}} \\
B_{0}^{1, l} & =\frac{2 m}{q} \sqrt{\left(\frac{2 m \eta_{c}^{2}}{(1+2 j)}\right)^{2}-\Omega^{2}}
\end{aligned}
$$

a constraint on the magnetic field $B_{0}^{1, l}$.

The ground state energy eigenvalues for $n=1$ is

$E_{1, l}= \pm \sqrt{k^{2}+q^{2}+m^{2}+2 m \tilde{\omega}_{1, l}\left(2+\sqrt{\frac{\left(l-\frac{q \Phi}{2 \pi}\right)^{2}}{\alpha^{2}}+\eta_{c}^{2}}\right)+2 m \omega_{c}^{1, l} \frac{\left(l-\frac{q \Phi}{2 \pi}\right)}{\alpha}+2 m \Omega}$

where $\omega_{1, l}$ is given by Eq. (42).

The corresponding radial wave-functions is

$$
\psi_{1, l}=\rho^{\sqrt{\frac{\left(l-\frac{q \Phi}{2 \pi}\right)^{2}}{\alpha^{2}}+\eta_{c}^{2}}} e^{-\frac{\rho^{2}}{2}}\left(c_{0}+c_{1} \rho\right)
$$

where

$$
c_{1}=\left[\frac{2 m \eta_{c}}{\sqrt{m \tilde{\omega}_{1, l}}\left(1+2 \sqrt{\frac{\left(l-\frac{q \Phi}{2 \pi}\right)^{2}}{\alpha^{2}}+\eta_{c}^{2}}\right)}\right] c_{0}
$$


Equations (43)-(45) corresponds to the energy level and eigenfunction for the radial mode $n=1$ of the quantum system subject to a Coulomb-type potential in the context of Kaluza-Klein theory.

In Ref. [47], authors solved KG-oscillator in the five-dimensional Minkowski space-time with a quantum flux subjected to a Coulomb-type potential in Kaluza-Klein theory (section 3). By comparing the eigenvalue and eigenfunction (43)-(45) with those results obtained in earlier, we have seen that the results presented here get modified due to the presence of a uniform magnetic field $B_{0}$ and the wedge parameter $\alpha$ whose values are in the ranges $0<\alpha \leq 1$ and break their degeneracy.

Similar to earlier case, the energy eigenvalue $E_{n, l}\left(\Phi+\Phi_{0}\right)=E_{n, l \mp \tau}(\Phi)$, where $\Phi_{0}= \pm \frac{2 \pi}{q} \tau$ with $\tau=0,1,2, \ldots$ is a periodic function of the geometric quantum phase. This dependence of the relativistic energy level on the geometric quantum phase gives rise to a relativistic analogue of the AharonovBohm effect for bound states.

\section{Case C : Interactions with Linear potential}

We consider $\eta_{c} \rightarrow 0$. Thus the linear scalar potential is given by

$$
S(r)=\eta_{L} r
$$

The linear potential have studied by many authors in the relativistic quantum mechanics [58, 59, 60, 61, 62].

The radial wave-equations Eq. (12) becomes

$$
\left[\frac{d^{2}}{d r^{2}}+\frac{1}{r} \frac{d}{d r}+\tilde{\lambda}-\frac{l_{0}^{2}}{r^{2}}-\omega^{2} r^{2}-b r\right] \psi(r)=0 .
$$

Introduce a new variable $\rho=\sqrt{\omega} r$, then the Eq. (47) becomes

$$
\left[\frac{d^{2}}{d \rho^{2}}+\frac{1}{\rho} \frac{d}{d \rho}+\frac{\tilde{\lambda}}{\omega}-\frac{l_{0}^{2}}{\rho^{2}}-\rho^{2}-\theta \rho\right] \psi(\rho)=0 .
$$


Let the possible solution to Ee. (48) is

$$
\psi=\rho^{\left|l_{0}\right|} e^{-\frac{1}{2}(\theta+\rho) \rho} H(\rho)
$$

Substituting Eq. (49) into the Eq. (48), we obtain

$$
H^{\prime \prime}(\rho)+\left[\frac{\left(1+2\left|l_{0}\right|\right)}{\rho}-\theta-2 \rho\right] H^{\prime}(\rho)+\left[-\frac{\frac{\theta}{2}\left(1+2\left|l_{0}\right|\right)}{\rho}+\Theta_{0}\right] H(\rho)=0
$$

where $\Theta_{0}=\frac{\tilde{\lambda}}{\omega}-2\left(1+\left|l_{0}\right|\right)+\frac{\theta^{2}}{4}$.

Equation (50) is the biconfluent Heun's differential equation [13, 14, 15, $34,53,54]$ and $H(\rho)$ is the Heun polynomials.

Substituting the power series solution (19) into the Eq. (50), we obtain the following recurrence relation for the coefficients:

$$
c_{n+2}=\frac{1}{(n+2)\left(n+2+2 l_{0}\right)}\left[\frac{\theta}{2}\left(2 n+3+2\left|l_{0}\right|\right) c_{n+1}-\left(\Theta_{0}-2 n\right) c_{n}\right] .
$$

And the various coefficients are

$$
\begin{aligned}
c_{1} & =\frac{\theta}{2} c_{0}, \\
c_{2} & =\frac{1}{4\left(1+l_{0}\right)}\left[\frac{\theta}{2}\left(3+2\left|l_{0}\right|\right) c_{1}-\Theta_{0} c_{0}\right] .
\end{aligned}
$$

The power series expansion (19) becomes a polynomial of degree $n$ by imposing two conditions [13, 14, 15, 34, 43, 44, 45]:

$$
c_{n+1}=0 \quad, \quad \Theta_{0}=2 n \quad(n=1,2,3,4, \ldots)
$$

By analyzing the condition $\Theta_{0}=2 n$, we get the following energy eigenvalues $E_{n, l}$ :

$E_{n, l}= \pm \sqrt{k^{2}+q^{2}+m^{2}+2 m \omega l_{0}+2 \omega\left(n+1+\left|l_{0}\right|\right)-\frac{m^{2} \eta_{L}^{2}}{\omega^{2}}++2 m \omega_{c} l_{0}+2 m \Omega}$ 
where $l_{0}=\frac{1}{\alpha}\left(l-\frac{q \Phi}{2 \pi}\right)$.

Equation (54) is the relativistic energy eigenvalues of a Klein-Gordon oscillator in the magnetic cosmic string background in the context of KaluzaKlein theory with a linear confining potential.

For the radial mode $n=1, c_{2}=0$ which implies

$$
\omega_{1, l}=\left[\frac{m^{2} \eta_{L}^{2}}{2}\left(3+2\left|l_{0}\right|\right)\right]^{\frac{1}{3}} .
$$

a constraint on the parameter $\omega_{1, l}$. Therefore, the magnetic field is given by

$$
\begin{aligned}
\omega_{c}^{1, l} & =\sqrt{\omega_{1, l}^{2}-\eta_{L}^{2}-m^{2} \Omega^{2}} \\
\Rightarrow B_{0}^{1, l} & =\frac{2 m}{q} \sqrt{\omega_{1, l}^{2}-\eta_{L}^{2}-m^{2} \Omega^{2}} \\
\Rightarrow & =\frac{2 m}{q} \sqrt{\left[\frac{m^{2} \eta_{L}^{2}}{2}\left(3+2\left|l_{0}\right|\right)\right]^{\frac{2}{3}}-\eta_{L}^{2}-m^{2} \Omega^{2}}
\end{aligned}
$$

Therefore the ground state energy level

$$
\begin{aligned}
E_{1, l}= & \pm\left\{k^{2}+q^{2}+m^{2}+2 m\left(\omega_{c}^{1, l}+\omega_{1, l}\right) \frac{\left(l-\frac{q \Phi}{2 \pi}\right)}{\alpha}\right. \\
& \left.+2 \omega_{1, l}\left(2+\frac{\left|l-\frac{q \Phi}{2 \pi}\right|}{\alpha}\right)-\frac{m^{2} \eta_{L}^{2}}{\omega_{1, l}^{2}}+m^{2} \Omega^{2}\right\}^{\frac{1}{2}}
\end{aligned}
$$

And the corresponding eigenfunction is

$$
\psi=\rho^{\left|\frac{l-\frac{q \Phi}{2 \pi}}{\alpha}\right|} e^{-\frac{1}{2}\left(\frac{2 m \eta_{c}}{\omega^{\frac{3}{2}}}+\rho\right) \rho}\left(c_{0}+c_{1} \rho\right)
$$

where

$$
c_{1}=\frac{2 m \eta_{c}}{2 \omega_{1, l}^{\frac{3}{2}}} c_{0} .
$$

Equation (57)-(59) corresponds to the relativistic energy level and eigenfunction for the radial mode $n=1$ of the system subject to a linear confining potential in Kaluza-Klein theory. 
In Ref. [47], authors solved KG-oscillator in the five-dimensional Minkowski space-time with a quantum flux subjected to a linear potential in KaluzaKlein theory (section 4). By comparing the present eigenvalue and eigenfunction (57)-(59) with those results obtained earlier, we have seen that the results presented here get modified due to the presence of a uniform magnetic field $B_{0}$ and the wedge parameter $\alpha$ whose values are in the ranges $0<\alpha \leq 1$ and break their degeneracy. Also in comparison to the result obtained in Ref. [14] (section 3, Case 1), our result presented here get modified due to the presence of magnetic field in the quantum system.

The energy eigenvalue $E_{n, l}\left(\Phi+\Phi_{0}\right)=E_{n, l \mp \tau}(\Phi)$, where $\Phi_{0}= \pm \frac{2 \pi}{q} \tau$ with $\tau=0,1,2, \ldots$ is a periodic function of the geometric quantum phase. This dependence of the relativistic energy level on the geometric quantum phase gives rise to a relativistic analogue of the Aharonov-Bohm effect for bound states.

\section{Case D : Interactions without potential}

In this case, we consider zero scalar potential, $S=0$. Therefore, the radial wave-equations Eq. (10) becomes

$$
\left[\frac{d^{2}}{d r^{2}}+\frac{1}{r} \frac{d}{d r}+\lambda_{0}-\frac{l_{0}^{2}}{r^{2}}-m^{2} \tilde{\omega}^{2} r^{2}\right] \psi(r)=0
$$

where

$$
\lambda_{0}=E^{2}-k^{2}-q^{2}-m^{2}-2 m \omega_{c} \frac{\left(l-\frac{q \Phi}{2 \pi}\right)}{\alpha}-2 m \Omega .
$$

Transforming to a new variable $\rho=m \tilde{\omega} r^{2}$ into the Eq. (60), we obtain [63]

$$
\left[\frac{d^{2}}{d \rho^{2}}+\frac{1}{\rho} \frac{d}{d \rho}+\frac{1}{\rho^{2}}\left(-\xi_{1} \rho^{2}+\xi_{2} \rho-\xi_{3}\right)\right] \psi(\rho)=0
$$

where

$$
\xi_{1}=\frac{1}{4} \quad, \quad \xi_{2}=\frac{\lambda_{0}}{4 m \tilde{\omega}} \quad, \quad \xi_{3}=\frac{l_{0}^{2}}{4}
$$


Therefore, the energy eigenvalues is given by

$E_{n, l}= \pm \sqrt{k^{2}+q^{2}+m^{2}+2 m \Omega+2 m \tilde{\omega}\left(2 n+1+\frac{\left|l-\frac{q \Phi}{2 \pi}\right|}{\alpha}\right)+2 m \omega_{c} \frac{\left(l-\frac{q \Phi}{2 \pi}\right)}{\alpha}}$

where $n=0,1,2, \ldots$

Equation (64) is the relativistic energy eigenvalues of a KG-oscillator under a uniform magnetic field in the magnetic cosmic string space-time using Kaluza-Klein theory.

The wave-functions is given by

$$
\psi_{n, l}(\rho)=|N|_{n, l} \rho^{\frac{\left|l-\frac{q \Phi}{2 \pi}\right|}{2 \alpha}} e^{-\frac{\rho}{2}} L_{n}^{\left(\frac{l-\frac{q \Phi}{2 \pi}}{\alpha}\right)}(\rho)
$$

where $|N|_{n, l}=\left(\frac{n !}{2\left(n+\frac{\left|l-\frac{q \Phi}{2 \pi}\right|}{\alpha}\right) !}\right)^{\frac{1}{2}}$ is the normalization constant and $L_{n}^{\left(\frac{l-\frac{q \Phi}{2 \pi}}{\alpha}\right)}(\rho)$ is the generalized Laguerre polynomials.

In Ref. [9], authors solved KG-oscillator in the five-dimensional magnetic cosmic string space-time using Kaluza-Klein theory. By comparing the eigenvalue (64) with those results obtained, we have seen that the result presented in this work get modified due to the presence of a uniform magnetic field $B_{0}$ which causes shifted the energy level.

\section{Conclusions}

In this work, we have investigated KG-oscillator with a uniform magnetic field and quantum flux in the five-dimensional cosmic string space-time subject to scalar potentials of various kind using Kaluza-Klein theory. In Case A, we have considered a Cornell-type scalar potential and obtained the energy eigenvalues (23). We have seen that the external magnetic field $B_{0}$ has allowed values determined by the quantum numbers of the system which gives us a quantum effect. By analysing the recurrence condition $c_{n+1}=0$, 
we have evaluated the ground state energy level (26), eigenfunction (27)(28) and the allowed values of the magnetic field $B_{0}(25)$ for the radial mode $n=1$ instead of $n=0$. This effect arises due to the presence of a Cornelltype potential in the quantum system. In Case $\mathbf{B}$, we have considered a Coulomb-type scalar potential and obtained the energy eigenvalues (40). For the radial mode $n=1$, we have evaluated the ground state energy level (43), the eigenfunction (44)-(45) and the allowed values of the magnetic field $B_{0}$ by (42) instead of $n=0$. Here also this effect arises due to the presence of a Coulomb-type potential in the quantum system. In Case $\mathbf{C}$, we have considered a linear confining potential and obtained the non-compact expression of the energy eigenvalues (54). Following the similar procedure, we have evaluated the ground state energy level (57), the wave-function (58)-(59), and the allowed values of the magnetic field $B_{0}$ by (56) for the radial mode $n=1$. In all the cases, we have observed that the results presented here modified in comparison to those results obtained in Ref. [47] due to the presence of a uniform magnetic field $B_{0}$ and the wedge parameter $\alpha$ present in the relativistic quantum system which causes shifted the energy level and as well as the wave-function. We have also observed a quantum effect due to the dependence of magnetic field $B_{0}^{n, l}$ on the quantum numbers of the relativistic system. We see that for zero oscillator frequency $\Omega \rightarrow 0$ and the wedge parameter $\alpha \rightarrow 1$, the energy eigenvalue and eigenfunction presented in Case $A$ - Case $C$ here reduces to the results obtained in Ref. [44]. Since in gravitation and cosmology, the values of the wedge parameter $\alpha$ are in the ranges $0<\alpha \leq 1$, thus, the presence of the wedge parameter as well as the oscillator frequency of the particle causes shifted the eigenvalue and eigenfunction for each radial mode. Also, we see that for zero oscillator frequency $\Omega \rightarrow 0$ of the scalar particle, the results obtained here reduces to those in Ref. [46]. In Case D, we have solved the KG-oscillator subject to a uniform magnetic field in the magnetic cosmic string space-time in the context of Kaluza-Klein theory without any potential. We have obtained the energy 
eigenvalues (64) and eigenfunction (65).

In all the cases, we have observed that the angular momentum number $l$ is shifted, $l \rightarrow l_{0}=\frac{1}{\alpha}\left(l-\frac{q \Phi}{2 \pi}\right)$, an effective angular quantum number. Therefore, the relativistic energy eigenvalue and the wave-function obtained here depends on the geometric quantum phase [30, 49] and is a periodic function of it. Thus, we have that, $E_{n, l}\left(\Phi+\Phi_{0}\right)=E_{n, l \mp \tau}(\Phi)$, where $\Phi_{0}=$ $\pm \frac{2 \pi}{q} \tau$ with $\tau=0,1,2, \ldots$. This dependence of the relativistic energy level on the geometric quantum phase gives rise to a relativistic analogue of the Aharonov-Bohm effect for bound states.

Quantum systems with scalar potential of various kinds have been investigated in several branches of physics both in relativistic and non-relativistic limit with many applications, such as, for example, semiconductor heterostructures, electronic properties of the semiconductors, quantum wells, wires and dots, quantum liquids, He clusters, quark-antiquark interaction, pionic atom etc. As we have introduced a wedge parameter $\alpha$ (cosmic string parameter) in the five-dimensional line-element, therefore, the studies of the quantum system with scalar potential of different kinds in the presence of a uniform magnetic field would give a better tools in experimental purpose in comparison to the those results obtained in Ref. [47].

\section{Conflict of Interests}

Author declares that there is no conflict of interests regarding publication this manuscript.

\section{Data Availability}

There is no data associated with this manuscript. 


\section{Contribution}

F. Ahmed has done whole part of the work.

\section{References}

[1] S. Bruce and P. Minning, Nuovo Cimento II 106 A, 711 (1993).

[2] V. V. Dvoeglazov, Nuovo Cimento II 107 A, 1413 (1994).

[3] M. Moshinsky, J. Phys. A : Math. Gen. 22, L817 (1989).

[4] A. Boumali and N. Messai, Can. J. Phys. 92, 1460 (2014).

[5] K. Bakke and C. Furtado, Ann. Phys. (N. Y.) 355, 48 (2015).

[6] R. L. L. Vitoria and K. Bakke, EPJ Plus 131, 36 (2016).

[7] Z. Wang, Z. Long, C. Long and M. Wu, EPJ Plus 130, 36 (2015).

[8] L. C. N. Santosa and C. C. Barros Jr., EPJ C 78, 13 (2018).

[9] J. Carvalho, A. M. de M. Carvalho, E. Cavalcante and C. Furtado, Eur. Phys. J. C (2016) $76: 365$.

[10] F. Ahmed, Int. J. Geom. Meths. Mod. Phys. 17, 2050138 (2020).

[11] F. Ahmed, Chin. J. Phys. 66, 587 (2020).

[12] F. Ahmed, EPL 130, 40003 (2020).

[13] F. Ahmed, Adv. High Energy Phys. 2020, 8107025 (2020).

[14] F. Ahmed, Eur. Phys. J. C 80, 211 (2020).

[15] F. Ahmed, Adv. High Energy Phys. 2020, 5691025 (2020).

[16] F. Ahmed, Eur. Phys. J. C 78, 598 (2018). 
[17] Th. Kaluza, Sitzungsber. Preuss. Akad. Wiss. Berlin (Math. Phys) K 1, 966 (1921); Int. J. Mod. Phys. D 27, 1870001 (2018).

[18] O. Klein, Magazine for Phys. 37, 895 (1926); Nature 118, 516 (1927).

[19] T. Muta, An introduction to Kaluza-Klein Theories, H. C. Lee (ed.), World Scientific publishing Co Pvt. Litd, Singapore (1984).

[20] A. M. de M. Carvalho, C. Satiro and F. Moraes, Eur. Phys. Lett. 80, 46002 (2007).

[21] C. Satiro and F. Moraes, Mod. Phys. Lett. A 20, 2561 (2005).

[22] C. Satiro and F. Moraes, Eur. Phys. J. E 20, 173 (2006).

[23] C. Satiro and F. Moraes, Eur. Phys. J. E 25, 425 (2008).

[24] M. Barriola and A. Vilenkin, Phys. Rev. Lett. 63, 341 (1989).

[25] H. Mukai, P. R. G. Fernandes, B. F. de Oliveira and G. S. Dias, Phys. Rev. E 75, 061704 (2007).

[26] F. Moraes, Braz. J. Phys. 30, 304 (2000).

[27] A. Bohm, A. Mostafazadeh, H. Koizumi, Q. Niu and J. Zwanziger, The Geometric Phase in Quantum Systems: Foundations, Mathematical Concepts and Applications in Molecular and Condensed Matter Physics, Springer-Verlag, New York (2003).

[28] M. Peskin and A. Tonomura, Lect. Notes. Phys. Vol. 340, Springer, Berlin, Germany (1989).

[29] V. B. Bezerra, J. Math. Phys. 30, 2895 (1989).

[30] Y. Aharonov and D. Bohm, Phys. Rev. 115, 485 (1959). 
[31] M. A. Anacleto, I. G. Salako, F. A. Brito and E. Passos, Phys. Rev. D 92, 125010 (2015).

[32] V. R. Khalilov, Eur. Phys. J. C (2014) 74 : 2708.

[33] C. Coste, F. Lund and M. Umeki, Phys. Rev. E 60, 4908 (1999).

[34] R. L. L. Vitória and K. Bakke, Int. J. Mod. Phys. D 27, 1850005 (2018).

[35] R. L. L. Vitória and K. Bakke, Eur. Phys. J. Plus (2018) 133 : 490.

[36] R. L. L. Vitória and K. Bakke, Eur. Phys. J. C (2018) 78 : 175.

[37] R. L. L. Vitória and K. Bakke, Gen. Relativ. Grav. (2016) 48 : 161.

[38] F. Ahmed, Adv. High Energy Phys. 2020, 4832010 (2020).

[39] F. Ahmed, Eur. Phys. J. Plus (2020) 135 : 588

[40] F. Ahmed, EPL 131, 30002 (2020).

[41] C. Furtado, F. Moraes and V. B. Bezerra, Phys. Rev. D 59, 107504 (1999).

[42] C. Furtado, V. B. Bezerra and F. Moraes, Mod. Phys. Lett A 15, 253 (2000).

[43] E. V. B. Leite, H. Belich and K. Bakke, Adv. High Energy Phys. 2015, 925846 (2015).

[44] E. V. B. Leite, H. Belich and R. L. L. Vitória, Adv. High Energy Phys. 2019, 6740360 (2019).

[45] E. V. B. Leite, R. L. L. Vitória and H. Belich, Mod. Phys. A 34, 1950319 (2019).

[46] F. Ahmed, Mod. Phys. Lett. A, DOI: 10.1142/S0217732321500048. 
[47] E. V. B. Leite, H. Belich and R. L. L. Vitória, Braz. J. Phys. 50, 744 (2020).

[48] A. Vilenkin, Phys. Rep. 121, 263 (1985).

[49] G. de A. Marques, C. Furtado, V. B. Bezerra and F. Moraes, J. Phys. A : Math Theor. 34, 5945 (2001).

[50] C. Furtado and F. Moraes, J. Phys. A : Math. Theor. 33, 5513 (2000).

[51] M. K. Bahar and F. Yasuk, Adv. High Energy Phys. 2013, 814985 (2013).

[52] C. Alexandrou, P. de Forcrand and O. Jahn, Nuclear Phys. B (Proc. Supp.) 119, 667 (2003).

[53] A. Ronveaux, Heun's Differential Equations, Oxford University Press, Oxford (1995).

[54] S. Y. Slavyanov and W. Lay, Special Functions: A Unified Theory Based in Singularities, Oxford University Press, New York (2000).

[55] G. B. Arfken and H. J. Weber, Mathematical Methods For Physicists, Elsevier Academic Pres, London (2005).

[56] A Vercin, Phys. Lett. B 260, 120 (1991).

[57] J. Myrhein, E. Halvorsen and A. Vercin, Phys. Lett. B 278, 171 (1992).

[58] A. L. C. de Oliveira and E. R. Bezerra de Mello, Class. Quantum Grav. 23, 5249 (2006).

[59] M. S. Cunha, C. R. Muniz, H. R. Christiansen and V. B. Bezerra, Eur. Phys. J. C (2016) $76: 512$.

[60] R. L. L. Vitória and H. Belich, Adv. High Energy Phys. 2019, 1248393 (2019). 
[61] R. L. L. Vitória and K. Bakke, Euro. Phys. J. Plus (2016) 131 : 36.

[62] R. L. L. Vitória, C. Furtado and K. Bakke, Ann. Phys. (N. Y.) 370, 128 (2016).

[63] A. F. Nikiforov and V. B. Uvarov, Special Functions of Mathematical Physics, Birkhaüser, Basel (1988). 